\title{
Modelo hidrogeológico conceptual de la cuenca baja del Río Ora (Nandayure, Guanacaste)
}

\author{
Catalina Vargas Quesada ${ }^{1}$ \& Federico Arellano Harting ${ }^{2}$ \\ 1. Estudiante de la Maestría académica en Hidrogeología y Manejo de Recursos Hídricos.Hídricos cava008@gmail. \\ com
}

2. Consultor privado, profesor en la Universidad de Costa Rica y director de la tesis federicoarellanoh@gmail.com

Recibido: 13 noviembre 2012

Aceptado: 31 enero 2013

\section{RESUMEN}

La zona de estudio se encuentra en la provincia de Guanacaste, corresponde con la cuenca baja del río Ora. Esta presenta un área aproximada de $37,28 \mathrm{~km}^{2}$. Geológicamente se encuentran litologías de origen volcánico, sedimentario y aluvional. Con base en el balance hídrico de suelos se determina que la recarga potencial es de $827.58 \mathrm{~mm} / \mathrm{año}$, la cual corresponde al $32 \%$ de la precipitación de la zona. El acuífero aluvional se encuentra compuesto por clastos limpios, semiconsolidados, presenta conductividades hidráulicas altas de hasta $8 \mathrm{~m} /$ día y espesores de hasta 30 metros. El acuífero volcánico se encuentra compuesto por las rocas basálticas fracturadas del Complejo de Nicoya, por lo general presenta una permeabilidad secundaria dada por las fisuras. Presenta distintas transmisividades en función del fracturamiento de las rocas, que varían de 2,38 a 193,70 m²/día. En las rocas basálticas se identificaron las lavas fracturadas, sanas, meteorizadas, densas y sin fracturar.

Palabras claves: balance hídrico, acuífero aluvional, acuífero volcánico.

\begin{abstract}
The study area is located in the province of Guanacaste, corresponds to the lower basin of Ora River. This presents an area of $37,28 \mathrm{~km}^{2}$ approximately. Lithologies are geologically volcanic, sedimentary and alluvial. Based on soil water balance is determined that the net recharge potential is 861.99 $\mathrm{mm} /$ year, which corresponds to $32 \%$ of rainfall in the area. The water level found in the alluvium aquifer corresponds to an alluvial, with 7212.79 transmisividadades $\mathrm{m}^{2} /$ day. The aquifer is composed of volcanic basalt rocks fractured Nicoya Complex. Usually given secondary permeate through the cracks. Has different transmissivities based fracturing of rocks, the high transmissivity of $193.70 \mathrm{~m}^{2} /$ day correspond to the fractured lavas, low transmissivities of $2.38 \mathrm{~m}^{2} /$ day, corresponding to the dense lava without fractures, whereas there a range
\end{abstract}

of 0.11 to $20 \mathrm{~m} / \mathrm{day}$, corresponding to the weathered and fractured basalt.

Key words: water balance, alluvium aquifer, volcanic aquifer.

\section{Introducción}

En la zona de Guanacaste, el abastecimiento de agua es uno de los factores más importantes que se deben de tomar en cuenta para la planificación de proyectos a desarrollar. Por lo que las instituciones encargadas de suministrar el recurso, tales como municipalidades, ASADAS y el Instituto de Acueductos y Alcantarillados deben buscar soluciones a la demanda actual y futura. La municipalidad de Nandayure, tiene interés de buscar nuevas fuentes subterráneas de abastecimiento de agua, protegerlas y administrarlas adecuadamente, logrando un equilibrio entre el desarrollo de la región, el suministro y la extracción de agua. Es por eso que se caracterizó el acuífero que se encuentra en la cuenca baja del río Ora, para conocer las características hidráulicas y determinar su potencial para el consumo de agua y la planificación del desarrollo de las actividades.

La cuenca baja del río Ora presenta un área aproximada de $37,23 \mathrm{~km}^{2}$ abarca los pueblos de Estrada, el Carmen y Camaronal. Administrativamente se ubica en los cantones de Hojancha (hacia el noroeste del río Ora) y Nandayure (hacia el sureste del río Ora), con los distritos de Puerto Carrillo y Zapotal respectivamente. Geográficamente se encuentra entre 
las coordenadas $203000-211000 \mathrm{~N} / 375000$ -386000 E de la hoja topográfica Cerro Azul, 1:50000, IGN. (Fig. 1)

El objetivo general del estudio es establecer el modelo hidrogeológico conceptual del acuífero en la cuenca baja del río Ora, determinar la cantidad de agua disponible en el acuífero y estimar el potencial de extracción de agua subterránea.

\section{Metodología}

Para desarrollar la presente investigación se siguió la siguiente metodología (cuadro 1)

Etapa 1: Recopilación de la información bibliográfica y procesamiento preliminar.

Esta etapa consistió en la recopilación de la información bibliográfica de trabajos anteriores que se hayan elaborado en la zona, como también la recopilación de datos de pozos existentes en la zona, datos climatológicos, caudales de los ríos, entre otros. Obtención de datos en el registro de pozos del SENARA, AyA, IMN.

Etapa 2: Trabajo de campo.

Esta etapa consistió en la realización de las giras, para la obtención de los datos, las cuales se incluyeron las siguientes actividades:

- Cartografiado geológico, de las formaciones rocosas y superficiales a escala 1:25000.

- Aforos diferenciales en los principales afluentes del río Ora.

- Toma de muestras de diferentes tipos de suelo, de las distintas unidades establecidas, se realizó análisis de granulometría, contenido de humedad, porosidad, densidad aparente, capacidad de campo y punto de marchites. Para la clasificación de suelos se utilizó el Sistema Unificado de Clasificación de Suelos (A.S.T.M, 1993).

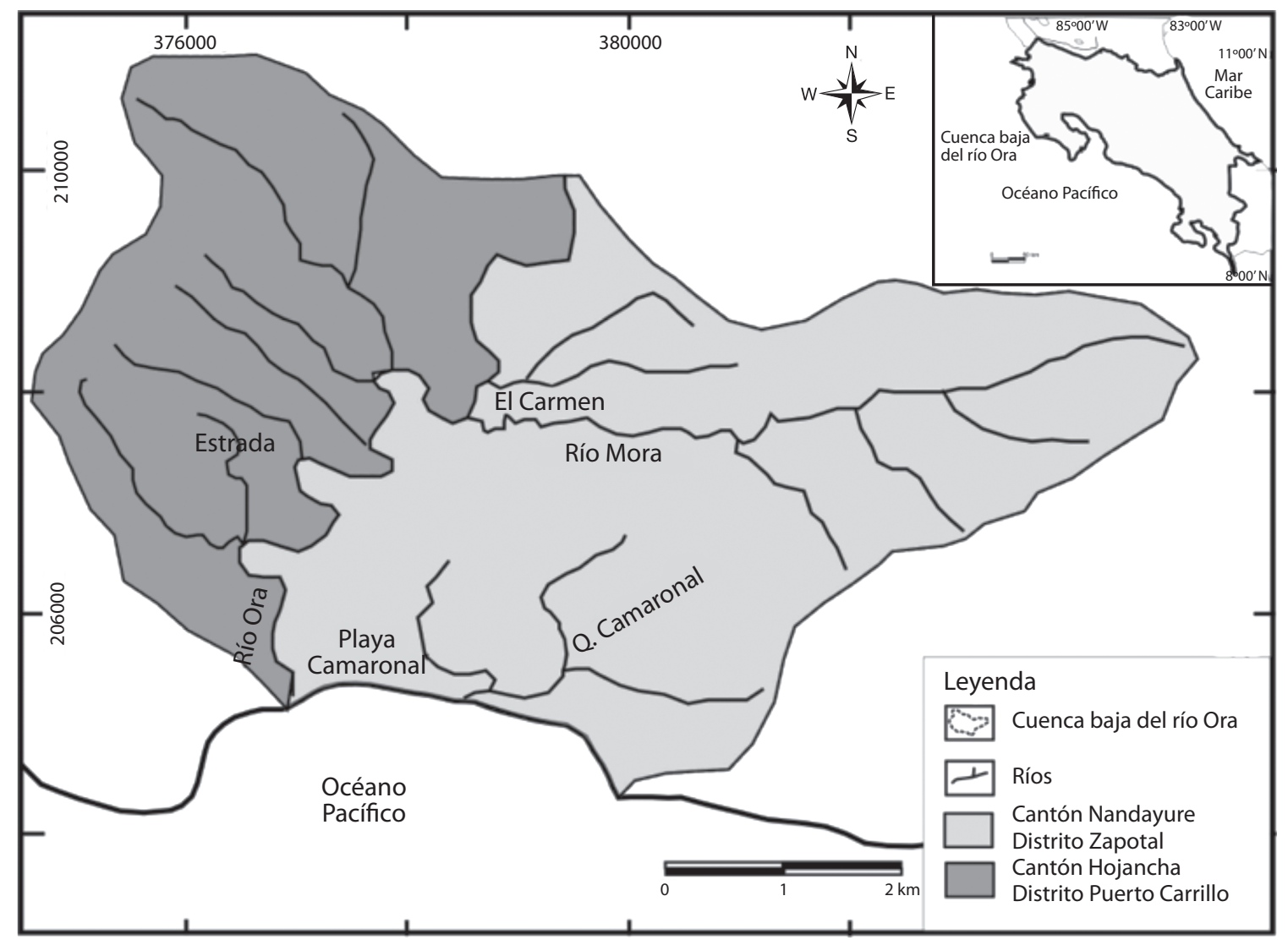

Figura 1: Mapa de ubicación de la zona de estudio. 
Metodología seguida en la presente investigación

\begin{tabular}{|c|c|c|}
\hline Etapa & Acciones realizadas & $\begin{array}{l}\text { Fecha que se realizó } \\
\text { la actividad }\end{array}$ \\
\hline Primera & Obtención de datos en el registro de pozos del SENARA, AyA, IMN. & Febrero 2010 \\
\hline \multirow[t]{7}{*}{ Segunda } & $\begin{array}{l}\text { Cartografiado geológico, de las formaciones rocosas y superficiales a escala } \\
1: 25000 \text {. }\end{array}$ & Marzo 2010 \\
\hline & Aforos diferenciales en los principales cursos de agua superficial & Abril 2010 \\
\hline & Toma de muestras de diferentes tipos de suelo & Mayo 2010 \\
\hline & $\begin{array}{l}\text { Pruebas de infiltración en las distintas unidades de suelos, utilizando el } \\
\text { método de doble anillo. }\end{array}$ & Mayo 2010 \\
\hline & $\begin{array}{l}\text { Prueba de bombeo en un pozo previamente establecidos para determinar } \\
\text { las características hidráulicas del acuífero. }\end{array}$ & Septiembre 2010 \\
\hline & $\begin{array}{l}\text { Determinar el uso actual del suelo, por medio de fotografías aéreas, } \\
\text { fotointerpretación, con corroboración de campo. }\end{array}$ & Mayo 2010 \\
\hline & Identificación de pozos excavados en la zona. & Agosto 2012 \\
\hline Tercera & $\begin{array}{l}\text { Se realizó el Balance de humedad de suelos para determinar la recarga neta } \\
\text { potencial (Schosinsky, \& Losilla, (2000) }\end{array}$ & Enero 2011-Agosto 2012 \\
\hline
\end{tabular}

- Pruebas de infiltración en las distintas unidades de suelos, se utilizó el método de doble anillo.

- Identificación de pozos excavados en la zona.

- Prueba de bombeo en un pozo previamente establecidos para determinar las características hidráulicas del acuífero.

- Determinar el uso actual del suelo, por medio de fotografías aéreas, fotointerpretación, con corroboración de campo.

Etapa 3: Interpretación y procesamiento final de los datos.

Esta etapa consistió en la integración e interpretación de los resultados obtenidos.

Se determinó la recarga neta potencial se utilizó el balance hídrico de suelos, desarrollado por Schosinsky \& Losilla, (2000), posteriormente se estimó la descarga natural y la descarga por extracción del agua subterránea por medio del registro de pozos del SENARA, como también de los pozos excavados. Con esta metodología se utilizó los datos de precipitación, evapotranspiración, infiltración, capacidad de campo, punto de marchites y características físicas de los suelos.

\section{Resultados}

Los resultados obtenidos se dividirán en cada sección establecida según la metodología utilizada.

\section{Resultados de Geología}

En el área de estudio se encontraron materiales de origen volcánico, sedimentario y aluvional. En las cuales se identificaron las siguientes unidades:

\section{Complejo de Nicoya}

En esta unidad se agrupan los materiales de origen volcánico, se encuentran a partir de los 60 m.s.n.m. Se identificaron rocas volcánicas basálticas las cuales constituyen los cerros de las zonas más elevadas. En su mayor parte las rocas se encuentran extremadamente meteorizadas y en un menor porcentaje con una leve meteorización. Son de color gris claro a negro cuando están sanas, mientras cuando se encuentran meteorizadas son de color café anaranjado y en su mayor parte producen suelos de color anaranjado.

Se observó la forma de lavas en almohadillas, las cuales se encuentran levemente sanas 
hacia el centro, mientras que hacia el borde se encuentra rodeada de arcilla. (Fig. 2)

\section{Formación Sabana Grande}

En la zona de estudio, las rocas sedimentarias se localizan en menor proporción que los basaltos, afloran por lo general en el cauce del rio Ora y al este de la cuenca. Son afloramientos puntuales y discontinuos. Litológicamente se caracterizan por presentarse en intercalaciones de lutitas y calcilutitas. Las calcilutitas son de color gris verdoso, en estratos decimétricos, las cuales presentan un contacto neto con las lutitas. Mientras que las lutitas son de color gris oscuro, son más friables y se presentan en estratos centimétricos.

\section{Aluviones cuaternarios del Río Ora}

Estos materiales han sido depositados por parte de los ríos Ora, Mora y los afluentes principales. Se encuentran en las partes más bajas, con elevaciones de 20 a $60 \mathrm{msnm}$.

Se observó en el campo que presentan estratos con granulometrías finas, de arenas finas a medias inconsolidados de color café claro, muestran espesores de hasta 1,5 m. Entre estos estratos se encontró un aluvión fino, con un espesor aproximado de $50 \mathrm{~cm}$, el cual se encuentra compuesto por clastos redondeados de tamaños centimétricos a milimétricos, formados de basaltos, areniscas y calcilutitas. Este espesor de aluvión se caracteriza por presentar un bajo porcentaje de matriz y se observa la diferencia de granulometria y textura entre el techo y la base del estrato, ya que en los bordes se encuentra con una granulometría más fina y un porcentaje mayor de arcillas. (Fig. 3)

\section{Resultados de hidrología}

De los resultados obtenidos de los aforos diferenciales se tiene que el río Lajas (al norte de cuenca), presenta variaciones en el caudal de 132,7 a 91,2 1/s, comportándose de manera influente en tramos de hasta de $3 \mathrm{~km}$ de longitud, el comportamiento varía de influencia a efluencia, para posteriormente definirse como efluente en la sección más baja de la cuenca.

El rio Ora presenta comportamiento de efluencia con caudales que varían de 142 a 166,2 $1 / \mathrm{s}$ en un tramo de $1,5 \mathrm{~km}$, seguidamente se da un comportamiento influente con caudales de 166,2 a 142,3 1/s. (Fig. 4)

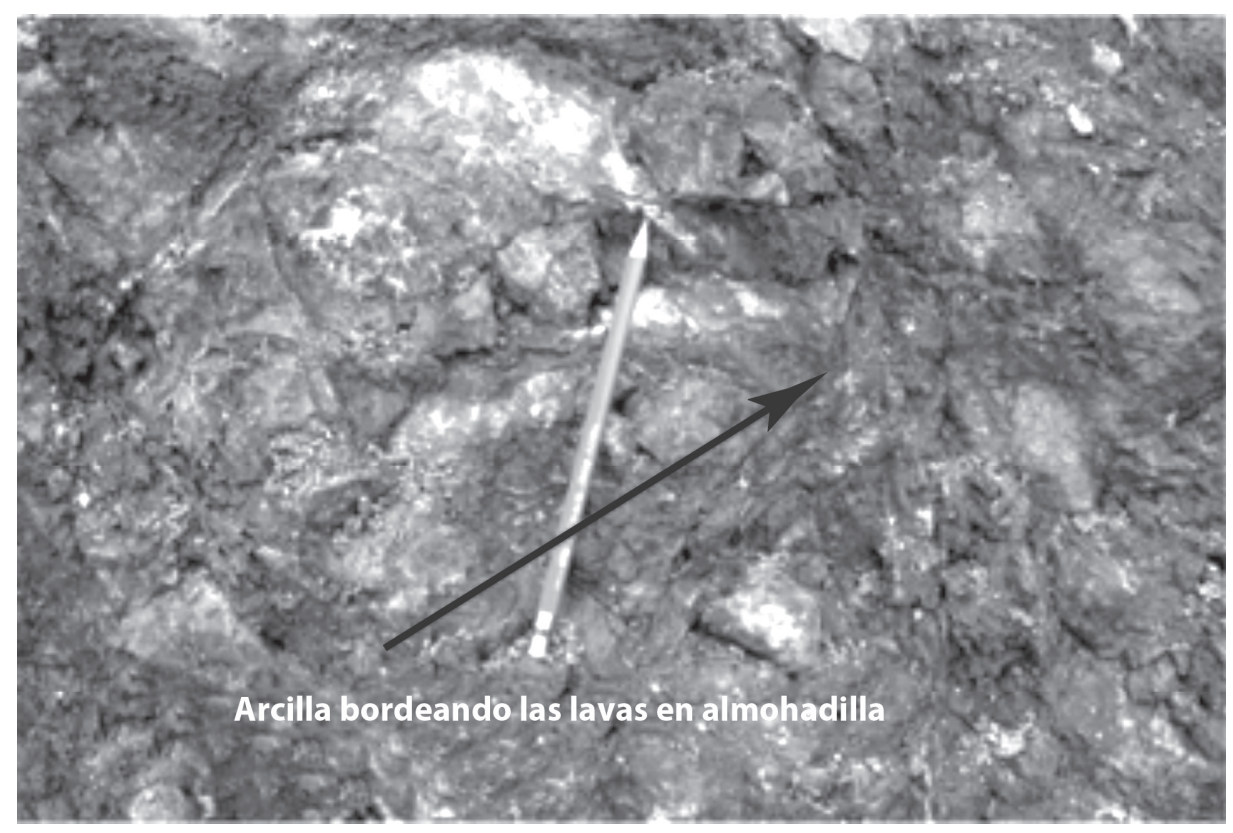

Figura 2: Lavas en almohadillas rodeadas de arcilla, provocando meteorización esferoidal. 


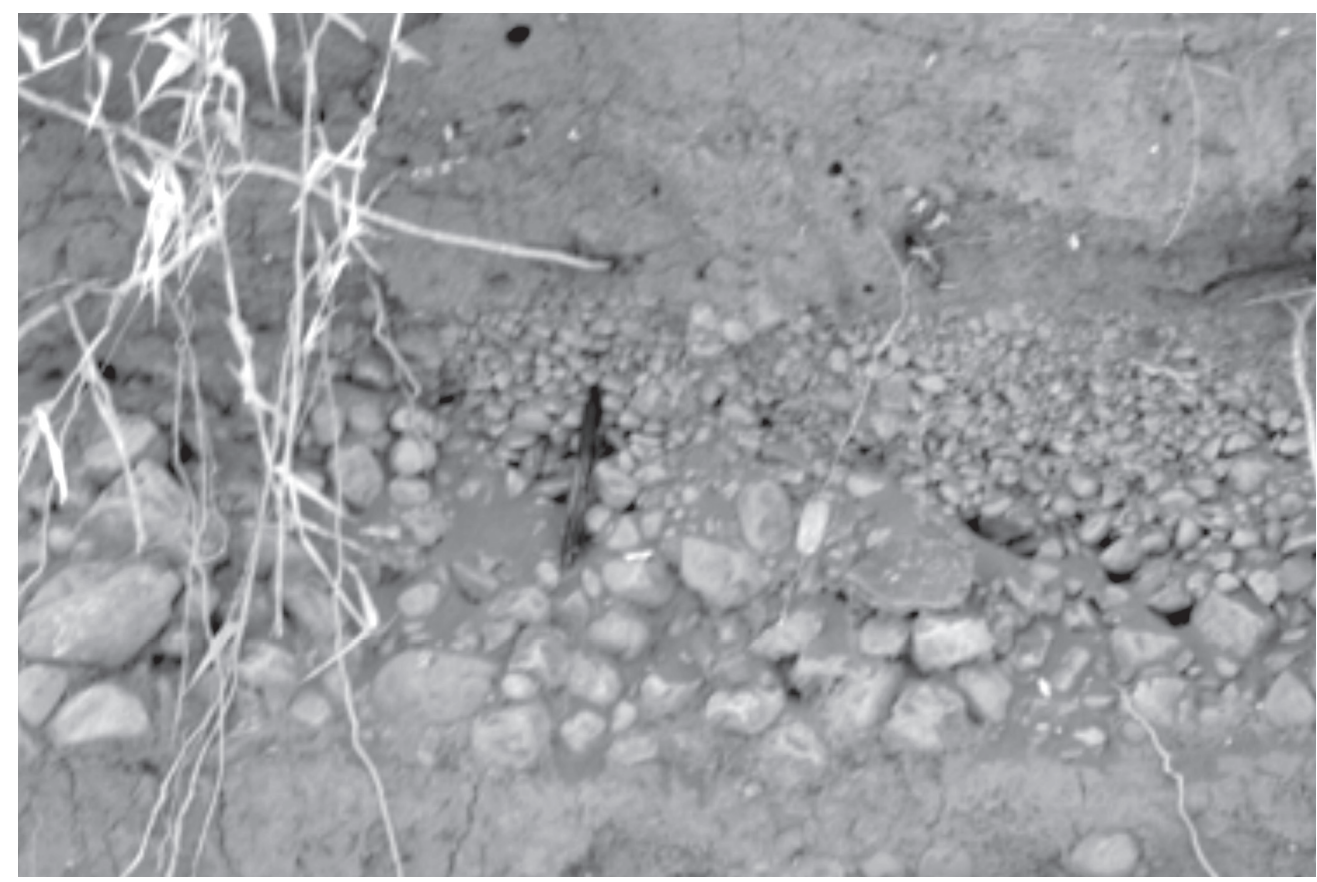

Figura 3: Estrato con poco contenido de arcilla, encontrado en las márgenes del río Ora, formado por clastos con gradación normal.

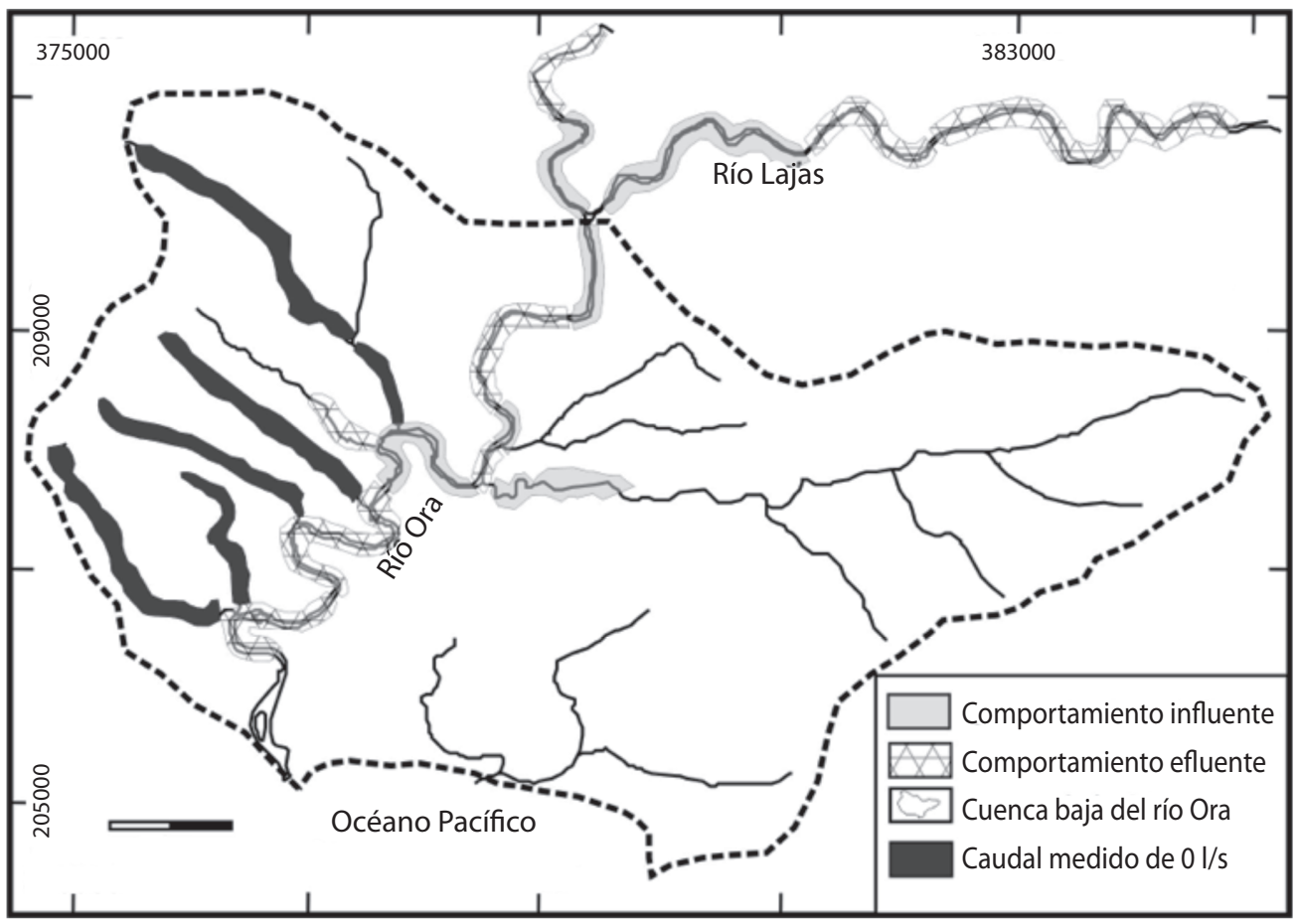

Figura 4: Mapa de influencia y efluencia de los ríos de la cuenca de estudio. 


\section{Resultados de uso actual de la tierra}

Las unidades identificadas son: área de bosque, por lo general se encuentran en las partes más altas de la zona, presenta mediana densidad de bosques y corresponde con 21,54 $\mathrm{km}^{2}$. En el área de terrenos reforestados predominan los cultivos de tecas, y se encuentran en gran parte de la cuenca, corresponden con el $6,75 \mathrm{~km}^{2}$, del área. El área urbana corresponde con las áreas ocupadas con infraestructura, abarca los poblados de Estrada, El Carmen y Camaronal, como también cementerios y carreteras. Estas zonas se encuentran desarrolladas en las partes bajas de la cuenca, específicamente en los aluviones. Corresponden con el $3,10 \mathrm{~km}^{2}$ del área total. Mientras que el área de pastos corresponde con el $5.84 \mathrm{~km}^{2}$ se presentan pastos, con poco desarrollo de ganadería.

Resultados de las pruebas de infiltración

Estas se realizaron en los suelos limos arcillosos con variaciones en el contenido de humedad, la textura y estructura del suelo. Existe una amplia variación de los resultados ya que van de 2.02 a $6454.23 \mathrm{~mm} /$ día, para analizar estos valores se procedió a clasificarlos según el uso del suelo y la geología de la zona definiendo las siguientes zonas: basalto con bosque, basalto con pasto, basalto con reforestación, aluvión con zona urbana, aluvión con pasto, aluvión con reforestación, lutitas-calcilutitas con bosque, lutitas- calcilutitas con reforestación y lutitas- calcilutitas con pasto. En la figura 5 se muestra un mapa con la ubicación de las mismas con el uso del suelo, mientras que en cuadro 2 se muestran los rangos establecidos según el tipo de uso de suelo.

\section{Resultados del balances de humedad de suelos}

Con base en el balance hídrico de suelos se determina que las recargas potenciales de las unidades varía de 745.49 a 1054.08 mm/año. La

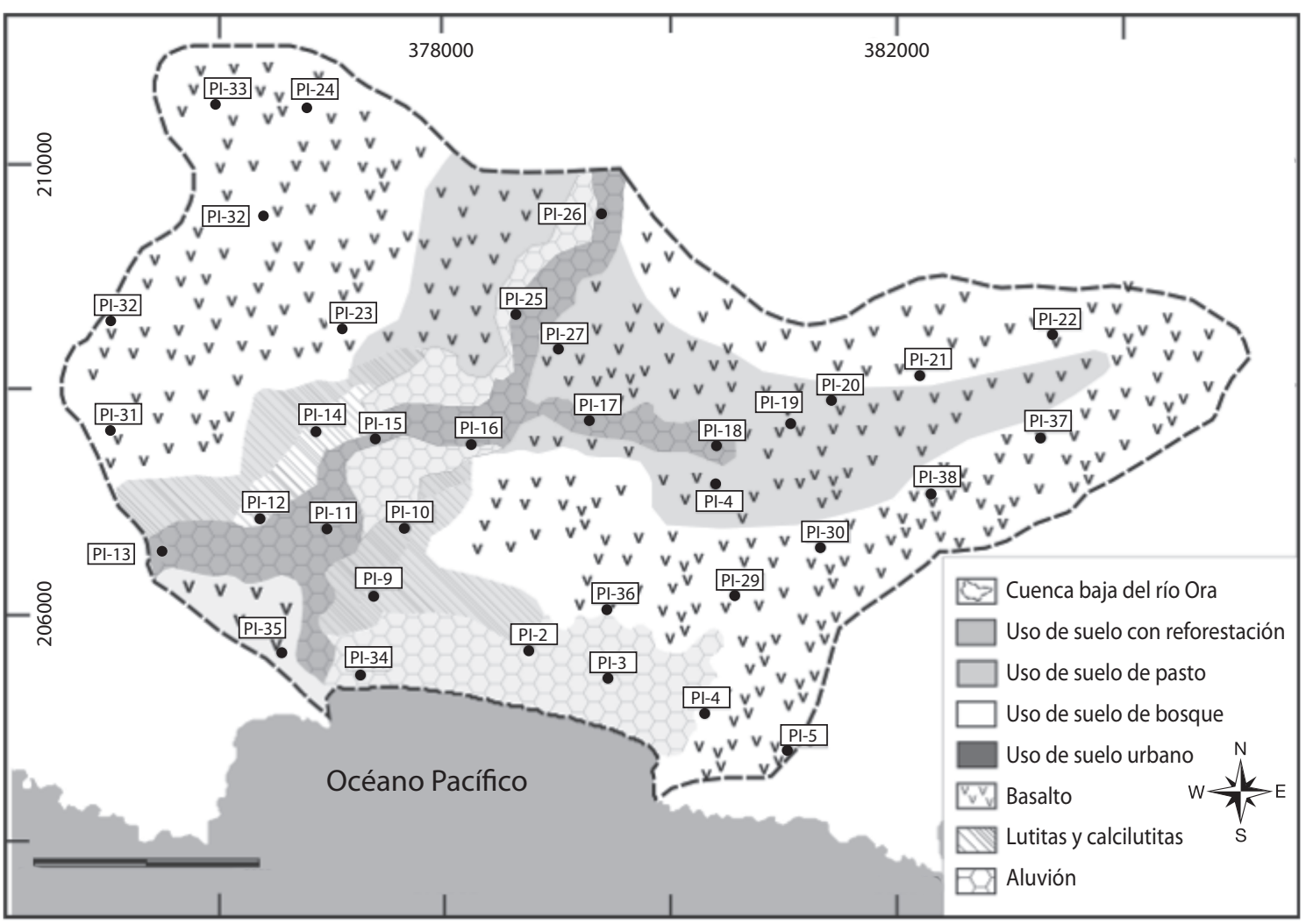

Figura 5: Mapa de ubicación de las pruebas de infiltración con respecto al uso de suelo y geología. 
CuAdRo 2

Rangos de capacidad de infiltración relacionadas al uso de la tierra.

\begin{tabular}{|c|c|c|c|c|c|c|c|c|c|}
\hline Prueba & Este & Norte & (mm/día) & Tipo de zona & Prueba & Este & Norte & (mm/día) & Tipo de zona \\
\hline 1 & 378135 & 206057 & 388,86 & Aluvión con zona urbana & 21 & 382320 & 208150 & 489,8 & Basalto con bosque \\
\hline 2 & 378885 & 205670 & 24,14 & Aluvión con pasto & 22 & 383556 & 208462 & 642,98 & Basalto con bosque \\
\hline 3 & 379578 & 205409 & 8,91 & Aluvión con pasto & 23 & 377176 & 208572 & 3265,51 & Basalto con bosque \\
\hline 4 & 380479 & 205174 & 210,11 & Basalto con reforestación & 24 & 376880 & 210538 & 185,83 & Basalto con bosque \\
\hline 5 & 381760 & 204840 & 7477,97 & Aluvión con zona urbana & 25 & 378761 & 208658 & 3,49 & Aluvión con reforestación \\
\hline 6 & 382350 & 204316 & 2,02 & Aluvión con zona urbana & 26 & 379520 & 209580 & 0,8 & Aluvión con zona urbana \\
\hline 7 & 382998 & 204856 & 13,26 & Aluvión con zona urbana & 27 & 379120 & 208361 & 2299,65 & Basalto con bosque \\
\hline 8 & 383274 & 204418 & 171,44 & Aluvión con zona urbana & 28 & 382393 & 205256 & 6459,23 & Aluvión con zona urbana \\
\hline 9 & 377472 & 206120 & 1294,9 & Lutitas y calcilutitas con pasto & 29 & 380702 & 206143 & 3436,63 & Basalto con bosque \\
\hline 10 & 377750 & 206750 & 266,39 & Lutitas y calcilutitass con reforestación & 30 & 381452 & 206556 & 2,86 & Basalto con bosque \\
\hline 11 & 377046 & 206750 & 229,85 & Aluvión con zona urbana & 31 & 375120 & 207625 & 848,08 & Basalto con bosque \\
\hline 12 & 376486 & 206848 & 3,17 & Lutitas y calcilutitas con bosque & 32 & 375133 & 208601 & 840.5 & Basalto con bosque \\
\hline 13 & 375327 & 206327 & 4,43 & Aluvión con zona urbana & 33 & 376065 & 210574 & 151,7 & Basalto con bosque \\
\hline 14 & 376981 & 207635 & 1699,44 & Lutitas y calcilutitas con bosque & 34 & 377350 & 205350 & 2.2 & Aluvión con pasto \\
\hline 15 & 377486 & 207543 & 116,06 & Aluvión con zona urbana & 35 & 386612 & 205500 & 1016,43 & Basalto con pasto \\
\hline 16 & 368360 & 207580 & 22,98 & Aluvión con zona urbana & 36 & 379585 & 206050 & 437,21 & Basalto con bosque \\
\hline 17 & 379390 & 207736 & 1710,72 & Aluvión con zona urbana & 37 & 383450 & 207670 & 642,98 & Basalto con bosque \\
\hline 18 & 380528 & 207464 & 1245,81 & Aluvión con zona urbana & 38 & 382450 & 207000 & 642,98 & Basalto con bosque \\
\hline 19 & 381210 & 207664 & 229,8 & Basalto con reforestación & 39 & 376500 & 209500 & 185,83 & Aluvión con zona urbana \\
\hline 20 & 381560 & 207885 & 655,5 & Basalto con reforestación & & & & & \\
\hline
\end{tabular}

recarga total al acuífero es de $827.58 \mathrm{~mm} / \mathrm{año}$. (Cuadro 3).

La unidad que presenta un mayor caudal de entrada corresponde con la zona boscosa con basalto con $455.05 \mathrm{l} / \mathrm{s}$, la unidad de basalto con pasto y basalto con cultivo es de $55.48 \mathrm{l} / \mathrm{s}$ y 148.01 $1 / \mathrm{s}$ respectivamente. Las lutitas-calcilutitas en zona de bosque y pasto presenta un caudal de 32.12 1/s y en la zona de cultivo 21 1/s. Mientras que los aluviones con pasto, zona urbana y con cultivo presentan caudales de $84.85 \mathrm{l} / \mathrm{s}, 74.7 \mathrm{l} / \mathrm{s}$, y $73.531 / \mathrm{s}$ respectivamente.

\section{Resultados de hidrogeología}

Hacia las partes bajas de la cuenca, específicamente en la parte aluvional del río Ora se presenta una alta extracción de agua subterránea por medio de pozos legales. A cada pozo se le determinó el uso y se le asignó un caudal de extracción, para posteriormente sumar los caudales. Realizando lo anterior se determina una extracción de 35.88 1/s, los cuales se desglosan de la siguiente manera. (Cuadro 4) (Fig. 6)

\section{Modelo Hidrogeológico Conceptual}

En la cuenca baja del rio Ora se definen dos niveles de agua:

- Uno en los aluviones en las partes bajas, que principalmente se recarga directamente por infiltración.

- Otro acuífero de origen volcánico, fisurado, que se recarga por percolación a través de las fracturas de las rocas.

El acuífero aluvional se encuentra compuesto por los materiales inconsolidados Cuaternarios, presenta lentes arcillosos y capas de clastos limpios. Muestra espesores de 8 metros, hacia playa Camaronal mientras que hacia la parte central de la cuenca baja del río Ora, se presentan los mayores espesores de hasta $30 \mathrm{~m}$. Corresponde con un acuífero libre, con un coeficiente de almacenamiento de 0.16 . El gradiente hidráulico varía de 0,033 a 0,058 con una dirección hacia el mar y hacia el río Ora principalmente. El movimiento del agua es a través de los 
CUADRO 3

Recargas netas de las unidades determinadas

\begin{tabular}{lcccccc}
\multicolumn{1}{c}{ Uso del suelo } & $\begin{array}{c}\text { Área } \\
\mathrm{km}^{2}\end{array}$ & $\begin{array}{c}\text { Recarga } \\
\mathrm{mm} / \mathrm{año}\end{array}$ & $\begin{array}{c}\text { Escorrentía } \\
\mathrm{mm} / \mathrm{año}\end{array}$ & $\begin{array}{c}\text { ETR } \\
\mathrm{mm} / \mathrm{año}\end{array}$ & $\begin{array}{c}\text { Retención } \\
\mathrm{mm} / \mathrm{año}\end{array}$ & $\begin{array}{c}\mathrm{P}=\mathrm{REC}+\mathrm{ESC} \\
+\mathrm{ETR}+\mathrm{RET}\end{array}$ \\
Basalto con Bosque & 19,25 & 745,49 & 0,00 & 1307 & 513,18 & 2565,67 \\
Basalto con Pasto & 1,70 & 1029,27 & 0,00 & 1226,93 & 309,69 & 2565,89 \\
Basalto con Cultivo & 6,10 & 765,19 & 263,61 & 1227,4 & 309,69 & 2565,89 \\
Aluvión con zona urbana & 2,30 & 1024,56 & 0,00 & 1231,56 & 309,62 & 2565,74 \\
Aluvión con pasto & 2,60 & 1029,27 & 0,00 & 1226,93 & 309,69 & 2565,89 \\
Aluvion con cultivo & 2,20 & 1054,08 & 0,00 & 1202,12 & 309,62 & 2565,82 \\
Lutitas y calcilutitas con bosque & 1,35 & 750,53 & 0,00 & 1302,18 & 513,18 & 2565,89 \\
Lutitas y calcilutitas con cultivo & 0,76 & 890,86 & 164,34 & 1201 & 309,69 & 2565,89 \\
Lutitas y calcilutitas con pasto & 0,98 & 1029,9 & 0,00 & 1226,9 & 309,69 & 2566,49 \\
Total & 37,24 & 827,58 & 46,53 & 1269,41 & 422,25 & 2565,78 \\
\hline
\end{tabular}

CuAdro 4

Caudales estimados para determinar la descarga artificial (SENARA, 2011)

\begin{tabular}{|c|c|c|c|c|c|c|c|}
\hline $\begin{array}{l}\mathrm{N} \text { de } \\
\text { pozos }\end{array}$ & uso & $\begin{array}{c}\text { Caudal } \\
\text { bombeo I/s }\end{array}$ & $\begin{array}{c}\text { Bombeo } \\
\text { (h/día) }\end{array}$ & meses & $\begin{array}{l}\text { Días al año } \\
\text { de bombeo }\end{array}$ & I/año & $\begin{array}{c}\text { Caudal anual } \\
\qquad 1 / 5\end{array}$ \\
\hline 37 & Doméstico & 136,9 & 8 & 12 & 365 & 38894,4 & 1,23 \\
\hline 1 & Abastecimiento público & 9,5 & 24 & 12 & 365 & 299592 & 9,5 \\
\hline 2 & Riego & 28 & 16 & 7 & 210 & 169344 & 5,36 \\
\hline 6 & Turístico & 36 & 12 & 12 & 365 & 94608 & 3 \\
\hline 2 & Industrial & 23,2 & 24 & 12 & 365 & 365817,6 & 11,6 \\
\hline 1 & Urbanístico & 4,5 & 24 & 12 & 365 & 141912 & 4,5 \\
\hline 1 & Domést - Riego & 0,5 & 12 & 12 & 365 & 7884 & 0,25 \\
\hline 6 & Varios & 7,2 & 6 & 12 & 365 & 9460,8 & 0,3 \\
\hline \multirow[t]{2}{*}{8} & Doméstico (Excavados) & 3,2 & 8 & 12 & 365 & 4204,8 & 0,13 \\
\hline & Total & & & & & 1131718 & 35,88 \\
\hline
\end{tabular}

clastos, presenta transmisividades que varían de 250 a $1200 \mathrm{~m}^{2} /$ día. Mientras que la permeabilidad del acuífero es de hasta $8 \mathrm{~m} /$ día.

El acuífero volcánico se encuentra compuesto por las rocas basálticas fracturadas del Complejo de Nicoya. Por lo general presenta una permeabilidad secundaria dada por las fisuras. Las fracturas que se presentan en las lavas muestran distintas direcciones, encontrándose con frecuencia fracturas rellenas de arcillas, que impiden el paso del agua, ocasionando que en esta zona existan variaciones de potencial de agua. Presenta distintas transmisividades en función del fracturamiento de las rocas. Corresponde a un acuífero volcánico, semiconfinado, con un gradiente hidráulico que varía de 0,05 a 0,09, con una dirección de las líneas de flujo hacia el sureste y noroeste. El valor de coeficiente de almacenamiento es de $2,7 \times 10^{-5}$ (Obtenido con base en las pruebas de bombeo analizadas)

\section{Discusión}

La recarga total de la cuenca es de 827.58 mm/año es decir $977.2 \mathrm{l} / \mathrm{s}$, correspondiente al $32 \%$ de la precipitación. Los valores finales para las unidades establecidas son semejantes pero se analizaron los resultados de cada unidad. La variación de las conductividades hidráulicas se debe al contenido de arcillas en el suelo 


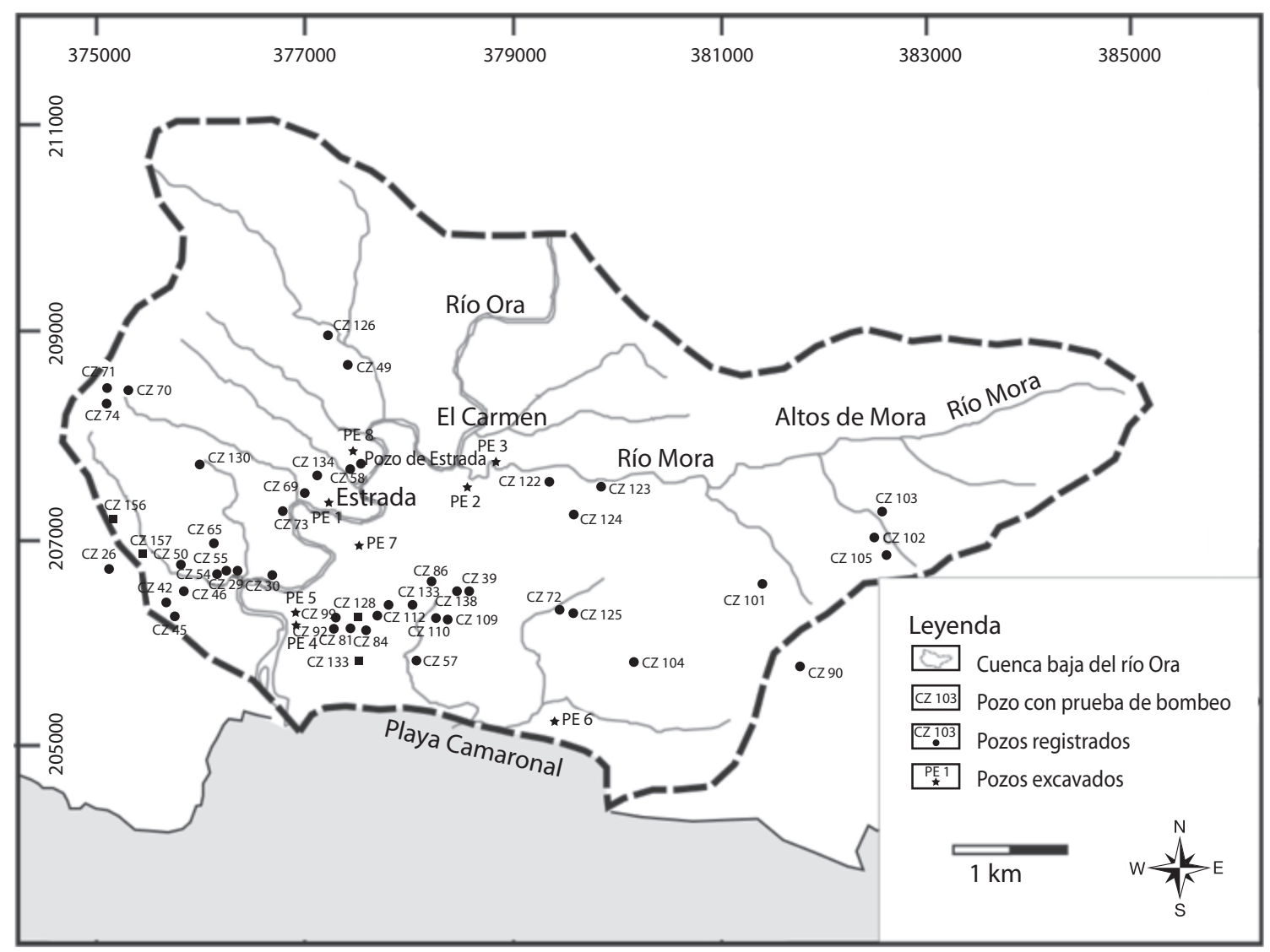

Figura 6: Mapa de ubicación de pozos legales y excavados de la cuenca de estudio.

producto de la meteorización de las rocas basálticas, como también a fracturas que se encuentren en los basaltos que provoquen que el agua se infiltre con mayor o menor velocidad. En la zona de aluvión con desarrollo urbano y pasto presenta valores promedios de $433.32 \mathrm{~mm} /$ día y el aluvión con reforestación con valores promedios de $420 \mathrm{~mm} /$ día. Los valores obtenidos representan litológicamente las variaciones que presenta el aluvión, la cual se encuentra compuesto por clastos con capas de arcillas y arenas intercaladas, donde las conductividades hidráulicas están en función de las capas arcillosas que se presenten. Otro aspecto que se debe de tomar en cuenta es que el aluvión al estar con zona urbana, lo compacta e impermeabiliza bajando su conductividad hidráulica. Mientas que las lutitas y calcilutitas con bosque y con zonas reforestadas los presentan valores promedios de 851.31 mm/día y $266.39 \mathrm{~mm} /$ día respectivamente Estas variaciones se interpretan como las fracturas y la estratificación de las rocas las cuales presentan distintas direcciones condicionando su valor.

Cabe menciona que estas diferencias de valores se deben a factores como la meteorización de las rocas tanto los basaltos, como los aluviones y el uso del suelo condiciona las características físicas como textura y estructura, por lo que se da unos valores semejantes entre cada uso y su ubicación.

Como ya se determinó la recarga del acuífero es principalmente por infiltración, la cual es 977,2 1/s. La recarga y la descarga en un ambiente de equilibrio deben de ser iguales. Mientras que la descarga a los ríos es de 293,52 1/s, la descarga al mar es de 381,91 1/s y la descarga por medio de pozos es de $35,81 / \mathrm{s}$. mientras que la descarga total es de $611.11 \mathrm{l} / \mathrm{s}$, dando una 
diferencia 50.39 1/s, esta diferencia entre un parámetro y otro es de $6 \%$, considerándose aceptable de acuerdo a la metodología aplicada.

Cabe mencionar que la mayor extracción de agua subterránea por medio de pozos se da hacia la parte central del río Ora específicamente en el acuífero aluvional. Este caudal se puede extraer para futuros pozos de abastecimiento y desarrollo siempre y cuando se cumplan las medidas de protección a cada pozo.

\section{Conclusiones}

Con base en el balance hídrico de suelos se determina que las recargas potenciales de las unidades varía de 751,4 a 1054.08 mm/año, mientras que la recarga total al acuífero es de $861.99 \mathrm{~mm} / \mathrm{año.}$

El acuífero aluvional se encuentra compuesto por los materiales inconsolidados Cuaternarios, presenta lentes arcillosos y capas de clastos, muestra espesores de 8 metros, hacia playa Camaronal y los mayores espesores hacia la parte central del río Ora, donde presenta un espesor de hasta $30 \mathrm{~m}$. Los niveles estáticos se encuentran someros, formando acuíferos libres.

El acuífero volcánico se encuentra compuesto por las rocas basálticas fracturadas del Complejo de Nicoya, presentan una permeabilidad secundaria dada por las fisuras. Las fractutras que se presentan en las lavas muestran distintas direcciones, encontrándose con frecuencia fracturas rellenas de arcillas, que impiden el paso del agua, ocasionando que en esta zona existan variaciones de potencial de agua.
La recarga al acuífero es de 977.2 1/s, mientras que la descarga es de 611.11 1/s, dando una diferencia 50.39 1/s, cabe mencionar que la mayor extracción de agua subterránea por medio de pozos se da hacia la parte central del río Ora específicamente en el acuífero aluvional. Este caudal se puede extraer para futuros pozos de abastecimiento y desarrollo siempre y cuando se cumplan las medidas de protección a cada pozo. Mientras que la extracción en el acuífero volcánico se encuentra condicionada por la permeabilidad secundaria dada por las fracturas de las rocas volcánicas.

\section{Referencias}

ASTM, 1993: Annual Book of ASTM Estándar. Section 4. Construction, Volumen 04.08 Soil and Rocks, geosyntheticcs.

CUSTODIO, E \& LLAMAS, M.R., 2001: Hidrologia subterránea.- 2350 págs. Editorial Omega, S.A., I, II, Barcelona, España

KUIJPERS, E.P., 1979: Geología del complejo Ofiolítico de Nicoya, Costa Rica.-Inf Semestral IGN., San José, Costa Rica (2) 15-75 del Água Subterrânea.- 115 pag. Banco Mundial, Washington, D.C.

SCHOSINSKY, G \& LOSILLA M., 2000: Modelo analítico para determinar la infiltración con base en la lluvia mensual.- Rev. Geológica de América Central, 23 : 43-55.

SENARA \& BGS, 1992: Mapa hidrogeológico de la Península de Nicoya, escala 1:200000. 